\title{
Maxwell's Equations on Cantor Sets: A Local Fractional Approach
}

\author{
Yang Zhao, ${ }^{1,2}$ Dumitru Baleanu, ${ }^{3,4,5}$ Carlo Cattani, ${ }^{6}$ De-Fu Cheng, ${ }^{1}$ and Xiao-Jun Yang ${ }^{7}$ \\ ${ }^{1}$ College of Instrumentation \& Electrical Engineering, Jilin University, Changchun 130061, China \\ ${ }^{2}$ Electronic and Information Technology Department, Jiangmen Polytechnic, Jiangmen 529090, China \\ ${ }^{3}$ Department of Chemical and Materials Engineering, Faculty of Engineering, King Abdulaziz University, P.O. Box 80204, \\ Jeddah 21589, Saudi Arabia \\ ${ }^{4}$ Department of Mathematics and Computer Sciences, Faculty of Arts and Sciences, Cankaya University, 06530 Ankara, Turkey \\ ${ }^{5}$ Institute of Space Sciences, Magurele, 077125 Bucharest, Romania \\ ${ }^{6}$ Department of Mathematics, University of Salerno, Via Ponte don Melillo, Fisciano, 84084 Salerno, Italy \\ ${ }^{7}$ Department of Mathematics and Mechanics, China University of Mining and Technology, Xuzhou Campus, Xuzhou, \\ Jiangsu 221008, China
}

Correspondence should be addressed to De-Fu Cheng; chengdefu@jlu.edu.cn

Received 3 October 2013; Accepted 7 November 2013

Academic Editor: Gongnan Xie

Copyright (C) 2013 Yang Zhao et al. This is an open access article distributed under the Creative Commons Attribution License, which permits unrestricted use, distribution, and reproduction in any medium, provided the original work is properly cited.

Maxwell's equations on Cantor sets are derived from the local fractional vector calculus. It is shown that Maxwell's equations on Cantor sets in a fractal bounded domain give efficiency and accuracy for describing the fractal electric and magnetic fields. Local fractional differential forms of Maxwell's equations on Cantor sets in the Cantorian and Cantor-type cylindrical coordinates are obtained. Maxwell's equations on Cantor set with local fractional operators are the first step towards a unified theory of Maxwell's equations for the dynamics of cold dark matter.

\section{Introduction}

Nondifferentiability, complexity, and similarity represent the basic properties of the nature. Fractals [1] are the basic characteristics of nature, which are that fractal geometry of substances generalizes to noninteger dimensions. Microphysics reveals the fractal behaviors of matter distribution in the universe [2] and soft materials [3].

Fractal time was used to describe the transport of charges and defects in the condensed matter [4]. In fractal space-time, the geometric analogue of relativistic quantum mechanics was presented in [5-8]. In fractal-Cantorian space-time $\Omega^{4 \alpha} \subset \Omega^{4}[9,10]$, the unified field theory, quantum physics, cosmology, and chaotic systems were discussed in [11-13].

Based on the fractal distribution of charged particles, the electric and magnetic fields in time-space $\Omega^{3 \alpha+1} \subset \Omega^{4}$ were developed in [14] and fractional Maxwell's equations were proposed in [15]. In $[16,17]$, the concept of static fractional electric potential was developed. Recently, based on the Hausdorff derivative, fractal continuum electrodynamics in time-space $\Omega^{4 \alpha} \subset \Omega^{4}$ was proposed [18]. The fractional differential form of Maxwell's equations on fractal sets was suggested in [19]. In [20], the Maxwell equations of fractional electrodynamics in time-space $\Omega^{3+\alpha} \subset \Omega^{4}$ were considered. The Maxwell equations on anisotropic fractal media in timespace $\Omega^{3 \alpha+1} \subset \Omega^{4}$ were developed in [21].

The local fractional calculus theory $[22,23]$ was applied to model some dynamics systems with nondifferentiable characteristics. In [22-26], the heat-conduction equation on Cantor sets was considered. In [27], the Navier-Stokes equations on Cantor sets based on local fractional vector calculus were proposed. Helmholtz and diffusion equations via local fractional vector calculus were reported in [28]. The Fokker-Planck equation with local fractional space derivative was suggested in [29]. In [30], Lagrangian and Hamiltonian 
mechanics with local fractional space derivative was presented. The measuring structures of time in fractal, fractional, classical, and discrete electrodynamics are shown in Figure 1.

The aim of this paper is to structure Maxwell's equations on Cantor sets from the local fractional calculus theory [23, $27,28]$ point of view. This paper is structured as follows. In Section 2, we introduce the basic definitions and theorems for local fractional vector calculus. In Section 3, Maxwell's equations on Cantor sets in the local fractional vector integral form are presented. Maxwell's equations on Cantor sets in the Cantorian and Cantor-type cylindrical coordinates are given in Section 4. Finally, Section 5 is devoted to conclusions.

\section{Fundaments}

In this section, we recall the basic definitions and theorems for local fractional vector calculus, which are used throughout the paper.

Local fractional gradient of the scale function $\phi$ is defined as $[23,27]$

$$
\nabla^{\alpha} \phi=\lim _{d V^{(\gamma)} \rightarrow 0}\left(\frac{1}{d V^{(\gamma)}} \oiint_{S^{(\beta)}} \phi d \mathbf{S}^{(\beta)}\right),
$$

where $\mathbf{S}^{(\beta)}$ is its bounding fractal surface, $V$ is a small fractal volume enclosing $P$, and the local fractional surface integral is given by $[23,27,28]$

$$
\iint u\left(r_{P}\right) d \mathbf{S}^{(\beta)}=\lim _{N \rightarrow \infty} \sum_{P=1}^{N} u\left(r_{P}\right) \mathbf{n}_{P} \Delta S_{P}^{(\beta)},
$$

with $N$ elements of area with a unit normal local fractional vector $\mathbf{n}_{P}, \Delta S_{P}^{(\beta)} \rightarrow 0$ as $N \rightarrow \infty$ for $\gamma=(3 / 2) \beta=3 \alpha$, and $\nabla^{\alpha}$ is denoted as the local fractional Laplace operator [22, 23].

The local fractional divergence of the vector function $\mathbf{u}$ is defined through [23]

$$
\nabla^{\alpha} \cdot \mathbf{u}=\lim _{d V^{(\gamma)} \rightarrow 0}\left(\frac{1}{d V^{(\gamma)}} \oiint_{S^{(2 \alpha)}} \mathbf{u} \cdot d \mathbf{S}^{(\beta)}\right),
$$

where the local fractional surface integral is suggested by [ 23 , $27,28]$

$$
\iint \mathbf{u}\left(r_{P}\right) \cdot d \mathbf{S}^{(\beta)}=\lim _{N \rightarrow \infty} \sum_{P=1}^{N} \mathbf{u}\left(r_{P}\right) \cdot \mathbf{n}_{P} \Delta S_{P}^{(\beta)},
$$

with $N$ elements of area with a unit normal local fractional vector $\mathbf{n}_{P}, \Delta S_{P}^{(\beta)} \rightarrow 0$ as $N \rightarrow \infty$ for $\gamma=(3 / 2) \beta=3 \alpha$.

The local fractional curl of the vector function $\mathbf{u}$ [23] is defined as follows:

$$
\nabla^{\alpha} \times \mathbf{u}=\lim _{d S^{(\beta)} \rightarrow 0}\left(\frac{1}{d S^{(\beta)}} \oint_{l^{(\alpha)}} \mathbf{u} \cdot d \mathbf{l}^{(\alpha)}\right) \mathbf{n}_{P}
$$

where the local fractional line integral of the function $\mathbf{u}$ along a fractal line $l^{\alpha}$ is given by [23]

$$
\begin{aligned}
\int_{l^{(\alpha)}} \mathbf{u} & \left(x_{P}, y_{P}, z_{P}\right) \cdot d \mathbf{l}^{(\alpha)} \\
& =\lim _{N \rightarrow \infty} \sum_{P=1}^{N} \mathbf{u}\left(x_{P}, y_{P}, z_{P}\right) \cdot \Delta \mathbf{l}_{P}^{(\alpha)},
\end{aligned}
$$

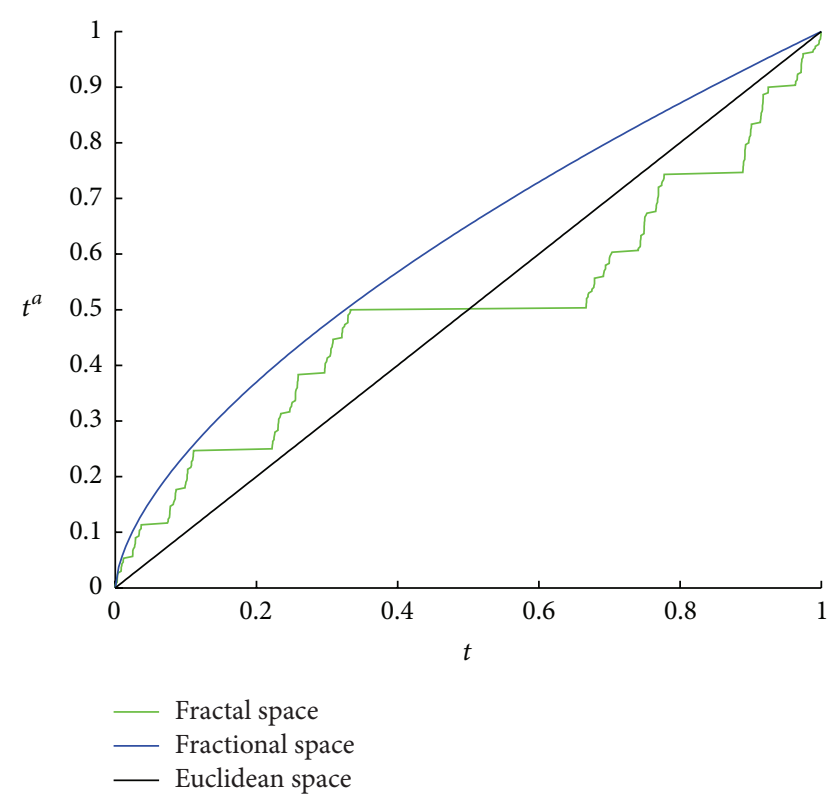

FIGURE 1: Graph for comparison of the measuring structures of time in fractal, fractional, and classical electrodynamics.

with the elements of line $\Delta \mathbf{l}_{P}^{(\alpha)}$ requiring that all $\left|\Delta l_{P}^{(\alpha)}\right| \rightarrow 0$ as $N \rightarrow \infty$ and $\beta=2 \alpha$.

The local fractional Gauss theorem of the fractal vector field states that $[23,27]$

$$
\iiint_{V^{(\gamma)}} \nabla^{\alpha} \cdot \mathbf{u} d V^{(\gamma)}=\oiint_{S^{(\beta)}} \mathbf{u} \cdot d \mathbf{S}^{(\beta)},
$$

where the local fractional volume integral of the function $\mathbf{u}$ is written as [23]

$$
\iiint \mathbf{u}\left(r_{P}\right) d V^{(\gamma)}=\lim _{N \rightarrow \infty} \sum_{P=1}^{N} \mathbf{u}\left(r_{P}\right) \Delta V_{P}^{(\gamma)}
$$

with the elements of volume $\Delta V_{P}^{(\gamma)} \rightarrow 0$ as $N \rightarrow \infty$ and $\gamma=(3 / 2) \beta=3 \alpha$.

The local fractional Stokes theorem of the fractal field states that $[23,27]$

$$
\oint_{l^{(\alpha)}} \mathbf{u} \cdot d \mathbf{l}^{(\alpha)}=\iint_{S^{(\beta)}}\left(\nabla^{\alpha} \times \mathbf{u}\right) \cdot d \mathbf{S}^{(\beta)} .
$$

The Reynolds transport theorem of the local fractional vector field states that [27]

$$
\begin{aligned}
& \frac{D^{\alpha}}{D t^{\alpha}} \iiint_{V^{(\gamma)}} F(x, t) d V^{(\gamma)} \\
& \quad=\iiint_{V^{(\gamma)}} \frac{\partial^{\alpha}}{\partial t^{\alpha}} F(x, t) d V^{(\gamma)}+\oiint_{S^{(\beta)}} F(x, t) \boldsymbol{v} \cdot d \mathbf{S}^{(\beta)},
\end{aligned}
$$

where $\boldsymbol{v}$ is the fractal fluid velocity.

\section{Local Fractional Integral Forms of Maxwell's Equations on Cantor Sets}

According to fractional complex transform method [31], the fact that the classical differential equations always transform 
into the local fractional differential equations leads to the idea of yielding Maxwell's equations on Cantor sets using the local fractional vector calculus.

3.1. Charge Conservations in Local Fractional Field. Let us consider the total charge, which is described as follows:

$$
Q=\iiint_{V^{(\gamma)}} \rho(r, t) d V^{(\gamma)},
$$

and the total electrical current is as follows

$$
I=\iint_{S^{(\beta)}} J(r, t) \cdot d \mathbf{S}^{(\beta)},
$$

where $\rho(r, t)$ is the fractal electric charge density and $J(r, t)$ is the fractal electric current density.

The Reynolds transport theorem in the fractal field gives

$$
\begin{aligned}
\frac{D^{\alpha} Q}{D t^{\alpha}} & =\frac{D^{\alpha}}{D t^{\alpha}} \iiint_{V^{(\gamma)}} \rho(r, t) d V^{(\gamma)} \\
& =\iiint_{V^{(\gamma)}} \frac{\partial^{\alpha} \rho(r, t)}{\partial t^{\alpha}} d V^{(\gamma)}+\oiint_{S^{(\beta)}} \rho(r, t) v \cdot d \mathbf{S}^{(\beta)} \\
& =0,
\end{aligned}
$$

which leads to

$$
\iiint_{V^{(\gamma)}} \frac{\partial^{\alpha} \rho(r, t)}{\partial t^{\alpha}} d V^{(\gamma)}+\oiint_{S^{(\beta)}} \rho(r, t) \boldsymbol{v} \cdot d \mathbf{S}^{(\beta)}=0 .
$$

From (14), we have

$$
\begin{aligned}
& \iiint_{V^{(\gamma)}} \frac{\partial^{\alpha} \rho(r, t)}{\partial t^{\alpha}} d V^{(\gamma)}+\oiint_{S^{(\beta)}} J(r, t) \cdot d \mathbf{S}^{(\beta)} \\
& \quad=\iiint_{V^{(\gamma)}}\left(\frac{\partial^{\alpha} \rho(r, t)}{\partial t^{\alpha}}+\nabla^{\alpha} \cdot J(r, t)\right) d V^{(\gamma)} \\
& \quad=0
\end{aligned}
$$

where $J=\rho \boldsymbol{v}$ represents the current density in the fractal field.

Hence, from (15), we get

$$
\frac{\partial^{\alpha} \rho(r, t)}{\partial t^{\alpha}}+\nabla^{\alpha} \cdot J(r, t)=0 .
$$

By analogy with electric charge density in the fractal field, we obtain the conservation of fractal magnetic charge, namely,

$$
\frac{\partial^{\alpha} \rho_{m}(r, t)}{\partial t^{\alpha}}+\nabla^{\alpha} \cdot J_{m}(r, t)=0
$$

where $\rho_{m}(r, t)$ is the fractal magnetic charge density and $J_{m}(r, t)$ is the fractal magnetic current density in the fractal field.

3.2. Formulation of Maxwell's Equations on Cantor Sets. We now derive Maxwell's equations on Cantor set based on the local fractional vector calculus.
3.2.1. Gauss's Law for the Fractal Electric Field. From (3), the electric charge density can be written as

$$
\nabla^{\alpha} \cdot D=\lim _{d V^{(\gamma)} \rightarrow 0}\left(\frac{1}{d V^{(\gamma)}} \oiint_{S^{(2 \alpha)}} D \cdot d \mathbf{S}^{(\beta)}\right)=\rho,
$$

where $D$ is electric displacement in the fractal electric field.

From (7), (18) becomes

$$
\begin{aligned}
& \iiint_{V^{(\gamma)}} \nabla^{\alpha} \cdot D d V^{(\gamma)}=\oiint_{S^{(\beta)}} D \cdot d \mathbf{S}^{(\beta)}, \\
& \iiint_{V^{(\gamma)}} \nabla^{\alpha} \cdot D d V^{(\gamma)}=\iiint_{V^{(\gamma)}} \rho d V^{(\gamma)} .
\end{aligned}
$$

Hence, we obtain Gauss's law for the fractal electric field in the form

$$
\oiint_{S^{(\beta)}} D \cdot d \mathbf{S}^{(\beta)}=\iiint_{V^{(\gamma)}} \rho d V^{(\gamma)} .
$$

3.2.2. Ampere's Law in the Fractal Magnetic Field. Mathematically, Ampere's law in the fractal magnetic field can be suggested as [23]

$$
\oint_{l^{(\alpha)}} H \cdot d \mathbf{l}^{(\alpha)}=I,
$$

where $H$ is the magnetic field strength in the fractal field.

The current density in the fractal field can be written as

$$
\nabla^{\alpha} \times H=\lim _{d S^{(\beta)} \rightarrow 0}\left(\frac{1}{d S^{(\beta)}} \oint_{l^{(\alpha)}} H \cdot d \mathbf{l}^{(\alpha)}\right) \mathbf{n}_{P}=J,
$$

which leads to

$$
\begin{aligned}
& \oint_{l^{(\alpha)}} H \cdot d \mathbf{l}^{(\alpha)}=\iint_{S^{(\beta)}}\left(\nabla^{\alpha} \times H\right) \cdot d \mathbf{S}^{(\beta)}, \\
& \iint_{S^{(\beta)}} J \cdot d \mathbf{S}^{(\beta)}=\iint_{S^{(\beta)}}\left(\nabla^{\alpha} \times H\right) \cdot d \mathbf{S}^{(\beta)} .
\end{aligned}
$$

The total current in the fractal fried reads

$$
I=\iint_{S^{(\beta)}}\left(J_{a}+J_{b}\right) \cdot d \mathbf{S}^{(\beta)},
$$

where $J_{a}$ is the conductive current and

$$
J_{b}=\frac{\partial^{\alpha} D}{\partial t^{\alpha}}
$$

which satisfies the following condition:

$$
\begin{aligned}
I_{b} & =\oiint_{S^{(\beta)}} J_{b} \cdot d \mathbf{S}^{(\beta)} \\
& =\oiint_{S^{(\beta)}} \frac{\partial^{\alpha}}{\partial t^{\alpha}} D \cdot d \mathbf{S}^{(\beta)} \\
& =\frac{\partial^{\alpha}}{\partial t^{\alpha}} \oiint_{S^{(\beta)}} D \cdot d \mathbf{S}^{(\beta)} .
\end{aligned}
$$

Hence, Ampere's law in the fractal field is expressed as follows:

$$
\oint_{l^{(\alpha)}} H \cdot d \mathbf{l}^{(\alpha)}=\iint_{S^{(\beta)}}\left(J_{a}+\frac{\partial^{\alpha} D}{\partial t^{\alpha}}\right) \cdot d \mathbf{S}^{(\beta)} .
$$


3.2.3. Faraday's Law in the Fractal Electric Field. Mathematically, Faraday's law in the local fractional field is expressed as

$$
E=-\frac{\partial^{\alpha} \varphi}{\partial t^{\alpha}}
$$

where $\varphi$ is the magnetic potential in the fractal field and $E$ is the electrical field strength in the fractal field.

From (28), we have

$$
\begin{aligned}
\nabla^{\alpha} \times E & =\lim _{d S^{(\beta)} \rightarrow 0}\left(\frac{1}{d S^{(\beta)}} \oint_{l^{(\alpha)}} E \cdot d \mathbf{l}^{(\alpha)}\right) \mathbf{n}_{P} \\
& =-\frac{\partial^{\alpha}}{\partial t^{\alpha}}\left(\nabla^{\alpha} \times \varphi\right)=-\frac{\partial^{\alpha} B}{\partial t^{\alpha}},
\end{aligned}
$$

where $B$ is the magnetic induction in the fractal field.

In view of (9), we rewrite (29) as

$$
\begin{gathered}
\oint_{l^{(\alpha)}} E \cdot d \mathbf{l}^{(\alpha)}=\iint_{S^{(\beta)}}\left(\nabla^{\alpha} \times E\right) \cdot d \mathbf{S}^{(\beta)}, \\
\iint_{S^{(\beta)}}\left(\nabla^{\alpha} \times E\right) \cdot d \mathbf{S}^{(\beta)}=\iint_{S^{(\beta)}}\left(-\frac{\partial^{\alpha} B}{\partial t^{\alpha}}\right) \cdot d \mathbf{S}^{(\beta)} \\
=-\frac{\partial^{\alpha}}{\partial t^{\alpha}} \iint_{S^{(\beta)}} B \cdot d \mathbf{S}^{(\beta)} .
\end{gathered}
$$

So, from (30), Faraday's law in the fractal field reads as

$$
\oint_{l^{(\alpha)}} E \cdot d \mathbf{l}^{(\alpha)}+\frac{\partial^{\alpha}}{\partial t^{\alpha}} \iint_{S^{(\beta)}} B \cdot d \mathbf{S}^{(\beta)}=0 .
$$

3.2.4. Magnetic Gauss's Law for the Fractal Magnetic Field. From (3), we derive the local fractional divergence of the magnetic induction in the fractal field, namely,

$$
\nabla^{\alpha} \cdot B=\lim _{d V^{(\gamma)} \rightarrow 0}\left(\frac{1}{d V^{(\gamma)}} \oiint_{S^{(2 \alpha)}} B \cdot d \mathbf{S}^{(\beta)}\right)=0 .
$$

Furthermore, the magnetic Gauss' law for the fractal magnetic field reads as

$$
\oiint_{S^{(\beta)}} B \cdot d \mathbf{S}^{(\beta)}=0
$$

3.2.5. The Constitutive Equations in the Fractal Field. Similar to the constitutive relations in fractal continuous medium mechanics [23], the constitutive relationships in fractal electromagnetic can be written as

$$
\begin{aligned}
& D=\varepsilon_{f} E, \\
& H=\mu_{f} B,
\end{aligned}
$$

where $\varepsilon_{f}$ is the fractal dielectric permittivity and $\mu_{f}$ is the fractal magnetic permeability.

\section{Local Fractional Differential Forms of Maxwell's Equations on Cantor Sets}

In this section, we investigate the local fractional differential forms of Maxwell's equations on Cantor sets.
The Cantor-type cylindrical coordinates can be written as follows [26]:

$$
\begin{gathered}
x^{\alpha}=R^{\alpha} \cos _{\alpha} \theta^{\alpha}, \\
y^{\alpha}=R^{\alpha} \sin _{\alpha} \theta^{\alpha}, \\
z^{\alpha}=z^{\alpha},
\end{gathered}
$$

with $R \in(0,+\infty), z \in(-\infty,+\infty), \theta \in(0, \pi]$, and $x^{2 \alpha}+y^{2 \alpha}=$ $R^{2 \alpha}$.

Making use of (35), we have

$$
\begin{aligned}
\nabla^{\alpha} \cdot \mathbf{r}= & \frac{\partial^{\alpha} r_{R}}{\partial R^{\alpha}}+\frac{1}{R^{\alpha}} \frac{\partial^{\alpha} r_{\theta}}{\partial \theta^{\alpha}}+\frac{r_{R}}{R^{\alpha}}+\frac{\partial^{\alpha} r_{z}}{\partial z^{\alpha}}, \\
\nabla^{\alpha} \times \mathbf{r}= & \left(\frac{1}{R^{\alpha}} \frac{\partial^{\alpha} r_{\theta}}{\partial \theta^{\alpha}}-\frac{\partial^{\alpha} r_{\theta}}{\partial z^{\alpha}}\right) \mathbf{e}_{R}^{\alpha} \\
& +\left(\frac{\partial^{\alpha} r_{R}}{\partial z^{\alpha}}-\frac{\partial^{\alpha} r_{z}}{\partial R^{\alpha}}\right) \mathbf{e}_{\theta}^{\alpha} \\
& +\left(\frac{\partial^{\alpha} r_{\theta}}{\partial R^{\alpha}}+\frac{r_{R}}{R^{\alpha}}-\frac{1}{R^{\alpha}} \frac{\partial^{\alpha} r_{R}}{\partial \theta^{\alpha}}\right) \mathbf{e}_{z}^{\alpha},
\end{aligned}
$$

where

$$
\begin{aligned}
\mathbf{r} & =R^{\alpha} \cos _{\alpha} \theta^{\alpha} \mathbf{e}_{1}^{\alpha}+R^{\alpha} \sin _{\alpha} \theta^{\alpha} \mathbf{e}_{2}^{\alpha}+z^{\alpha} \mathbf{e}_{3}^{\alpha} \\
& =r_{R} \mathbf{e}_{R}^{\alpha}+r_{\theta} \mathbf{e}_{\theta}^{\alpha}+r_{z} \mathbf{e}_{z}^{\alpha} .
\end{aligned}
$$

From (7) and (20), the local fractional differential form of Gauss's law for the fractal electric field is expressed by

$$
\nabla^{\alpha} \cdot D=\rho
$$

which leads to

$$
\frac{\partial^{\alpha} D_{R}}{\partial R^{\alpha}}+\frac{1}{R^{\alpha}} \frac{\partial^{\alpha} D_{\theta}}{\partial \theta^{\alpha}}+\frac{D_{R}}{R^{\alpha}}+\frac{\partial^{\alpha} D_{z}}{\partial z^{\alpha}}=\rho(R, \theta, z, t),
$$

where $D=D_{R} \mathbf{e}_{R}^{\alpha}+D_{\theta} \mathbf{e}_{\theta}^{\alpha}+D_{z} \mathbf{e}_{z}^{\alpha}$.

In view of (9) and (27), we can present the local fractional differential forms of Ampere's law in the fractal magnetic field

$$
\begin{gathered}
\nabla^{\alpha} \times H=J_{a}+\frac{\partial^{\alpha} D}{\partial t^{\alpha}} \\
\left(\frac{1}{R^{\alpha}} \frac{\partial^{\alpha} H_{\theta}}{\partial \theta^{\alpha}}-\frac{\partial^{\alpha} H_{\theta}}{\partial z^{\alpha}}\right) \mathbf{e}_{R}^{\alpha}+\left(\frac{\partial^{\alpha} H_{R}}{\partial z^{\alpha}}-\frac{\partial^{\alpha} H_{z}}{\partial R^{\alpha}}\right) \mathbf{e}_{\theta}^{\alpha} \\
+\left(\frac{\partial^{\alpha} H_{\theta}}{\partial R^{\alpha}}+\frac{H_{R}}{R^{\alpha}}-\frac{1}{R^{\alpha}} \frac{\partial^{\alpha} H_{R}}{\partial \theta^{\alpha}}\right) \mathbf{e}_{z}^{\alpha} \\
=J_{a}(R, \theta, z, t)+\frac{\partial^{\alpha} D(R, \theta, z, t)}{\partial t^{\alpha}}
\end{gathered}
$$

where $H=H_{R} \mathbf{e}_{R}^{\alpha}+H_{\theta} \mathbf{e}_{\theta}^{\alpha}+H_{z} \mathbf{e}_{z}^{\alpha}$. 
Using (9) and (31), the local fractional differential forms of Faraday's law in the fractal electric field can be written as

$$
\begin{gathered}
\nabla^{\alpha} \times E=-\frac{\partial^{\alpha} B}{\partial t^{\alpha}} \\
\left(\frac{1}{R^{\alpha}} \frac{\partial^{\alpha} E_{\theta}}{\partial \theta^{\alpha}}-\frac{\partial^{\alpha} E_{\theta}}{\partial z^{\alpha}}\right) \mathbf{e}_{R}^{\alpha}+\left(\frac{\partial^{\alpha} E_{R}}{\partial z^{\alpha}}-\frac{\partial^{\alpha} E_{z}}{\partial R^{\alpha}}\right) \mathbf{e}_{\theta}^{\alpha} \\
+\left(\frac{\partial^{\alpha} E_{\theta}}{\partial R^{\alpha}}+\frac{E_{R}}{R^{\alpha}}-\frac{1}{R^{\alpha}} \frac{\partial^{\alpha} E_{R}}{\partial \theta^{\alpha}}\right) \mathbf{e}_{z}^{\alpha} \\
=-\frac{\partial^{\alpha} B(R, \theta, z, t)}{\partial t^{\alpha}}
\end{gathered}
$$

where $E=E_{R} \mathbf{e}_{R}^{\alpha}+E_{\theta} \mathbf{e}_{\theta}^{\alpha}+E_{z} \mathbf{e}_{z}^{\alpha}$.

From (7) and (33), the local fractional differential forms of the magnetic Gauss law for the fractal magnetic field read as follows:

$$
\begin{gathered}
\nabla^{\alpha} \cdot B=0, \\
\frac{\partial^{\alpha} B_{R}}{\partial R^{\alpha}}+\frac{1}{R^{\alpha}} \frac{\partial^{\alpha} B_{\theta}}{\partial \theta^{\alpha}}+\frac{B_{R}}{R^{\alpha}}+\frac{\partial^{\alpha} B_{z}}{\partial z^{\alpha}}=0,
\end{gathered}
$$

where $B=B_{R} \mathbf{e}_{R}^{\alpha}+B_{\theta} \mathbf{e}_{\theta}^{\alpha}+B_{z} \mathbf{e}_{z}^{\alpha}$.

\section{Conclusions}

In this work, we proposed the local fractional approach for Maxwell's equations on Cantor sets based on the local fractional vector calculus. Employing the local fractional divergence and curl of the vector function, we deduced Maxwell's equations on Cantor sets. The local fractional differential forms of Maxwell's equations on Cantor sets in the Cantorian and Cantor-type cylindrical coordinates were discussed. Finding a formulation of Maxwell's equations on Cantor set within local fractional operators is the first step towards generalizing a simple field equation which allows the unification of Maxwell's equations to the standard model with the dynamics of cold dark matter. We noticed that the classical case was debated in [32].

\section{Conflict of Interests}

The authors declare that they have no conflict of interests regarding the publication of this paper.

\section{References}

[1] B. B. Mandelbrot, The Fractal Geometry of Nature, W. H. Freeman, New York, NY, USA, 1982.

[2] A. Aharony and J. Feder, Fractals in Physics, North-Holland, Amsterdam, The Netherlands, 990.

[3] H. P. Xie, Fractals in Rock Mechanics, Balkema, Rotterdam, The Netherlands, 1993.

[4] M. F. Shlesinger, "Fractal time in condensed matter," Annual Review of Physical Chemistry, vol. 39, no. 1, pp. 269-290, 1988.

[5] L. Nottale, "Fractals and the quantum theory of spacetime," International Journal of Modern Physics A, vol. 4, no. 19, pp. 5047-5117, 1989.
[6] L. Nottale, Fractal Space-Time and Microphysics: Towards a Theory of Scale Relativity, World Scientific, River Edge, NJ, USA, 1993.

[7] L. Nottale, "Scale relativity, fractal space-time and quantum mechanics," Chaos, Solitons and Fractals, vol. 4, no. 3, pp. 361388, 1994.

[8] G. N. Ord, "Fractal space-time: a geometric analogue of relativistic quantum mechanics," Journal of Physics A, vol. 16, no. 9, pp. 1869-1884, 1983.

[9] O. Zmeskal, M. Vala, M. Weiter, and P. Stefkova, "Fractalcantorian geometry of space-time," Chaos, Solitons \& Fractals, vol. 42, no. 3, pp. 1878-1892, 2009.

[10] L. Marek-Crnjac, "A short history of fractal-cantorian spacetime," Chaos, Solitons \& Fractals, vol. 41, no. 5, pp. 2697-2705, 2009.

[11] E. Goldfain, "Local scale invariance, cantorian space-time and unified field theory," Chaos, Solitons \& Fractals, vol. 23, no. 3, pp. 701-710, 2005.

[12] L. Nottale, "Scale relativity and fractal space-time: applications to quantum physics, cosmology and chaotic systems," Chaos, Solitons \& Fractals, vol. 7, no. 6, pp. 877-938, 1996.

[13] E. Goldfain, "Complexity in quantum field theory and physics beyond the standard model," Chaos, Solitons \& Fractals, vol. 28, no. 4, pp. 913-922, 2006.

[14] V. E. Tarasov, "Electromagnetic fields on fractals," Modern Physics Letters A, vol. 21, no. 20, pp. 1587-1600, 2006.

[15] V. E. Tarasov, "Fractional vector calculus and fractional Maxwell's equations," Annals of Physics, vol. 323, no. 11, pp. 2756-2778, 2008.

[16] J. A. Tenreiro Machado, I. S. Jesus, A. Galhano, and J. Boaventura Cunha, "Fractional order electromagnetic," Signal Processing, vol. 86, no. 10, pp. 2637-2644, 2006.

[17] I. S. Jesus and J. A. Machado, "Implementation of fractionalorder electromagnetic potential through a genetic algorithm," Communications in Nonlinear Science and Numerical Simulation, vol. 14, no. 5, pp. 1838-1843, 2009.

[18] A. S. Balankin, B. Mena, J. Patiño, and D. Morales, "Electromagnetic fields in fractal continua," Physics Letters A, vol. 377, no. 10-11, pp. 783-788, 2013.

[19] A. K. Golmankhaneh, A. K. Golmankhaneh, and D. Baleanu, "About Maxwell's equations on fractal subsets of $\mathbb{R}^{3}$," Central European Journal of Physics, vol. 11, no. 6, pp. 863-867, 2013.

[20] H. Nasrolahpour, "A note on fractional electrodynamics," Communications in Nonlinear Science and Numerical Simulation, vol. 18, no. 9, pp. 2589-2593, 2013.

[21] M. Ostoja-Starzewski, "Electromagnetism on anisotropic fractal media," Zeitschrift für Angewandte Mathematik und Physik, vol. 64, no. 2, pp. 381-390, 2013.

[22] X.-J. Yang, Local Fractional Functional Analysis and Its Applications, Asian Academic, Hong Kong, 2011.

[23] X.-J. Yang, Advanced Local Fractional Calculus and Its Applications, World Science, New York, NY, USA, 2012.

[24] C. F. Liu, S. S. Kong, and S. J. Yuan, "Reconstructive schemes for variational iteration method within Yang-Laplace transform with application to fractal heat conduction problem," Thermal Science, vol. 17, no. 3, pp. 715-721, 2013.

[25] A. M. Yang, Y. Z. Zhang, and Y. Long, "The Yang-Fourier transforms to heat-conduction in a semi-infinite fractal bar," Thermal Science, vol. 17, no. 3, pp. 707-713, 2013. 
[26] X.-J. Yang, H. M. Srivastava, J.-H. He, and D. Baleanu, "Cantortype cylindrical-coordinate method for differential equations with local fractional derivatives," Physics Letters A, vol. 377, no. 28-30, pp. 1696-1700, 2013.

[27] X.-J. Yang, D. Baleanu, and J. A. Tenreiro Machado, "Systems of Navier-Stokes equations on Cantor sets," Mathematical Problems in Engineering, vol. 2013, Article ID 769724, 8 pages, 2013.

[28] Y. J. Hao, H. M. Srivastava, H. Jafari, and X. J. Yang, "Helmholtz and diffusion equations associated with local fractional derivative operators involving the Cantorian and Cantor-type cylindrical coordinates," Advances in Mathematical Physics, vol. 2013, Article ID 754248, 5 pages, 2013.

[29] K. M. Kolwankar and A. D. Gangal, "Local fractional FokkerPlanck equation," Physical Review Letters, vol. 80, no. 2, pp. 214217, 1998.

[30] A. K. Golmankhaneh, A. K. Golmankhaneh, and D. Baleanu, "Lagrangian and Hamiltonian mechanics on fractals subset of real-line," International Journal of Theoretical Physics, vol. 52, no. 11, pp. 4210-4217, 2013.

[31] W. H. Su, X. J. Yang, H. Jafari, and D. Baleanu, "Fractional complex transform method for wave equations on Cantor sets within local fractional differential operator," Advances in Difference Equations, vol. 2013, p. 97, 2013.

[32] W. Krolikowski, "On Maxwell's equations unified with the dynamics of cold dark matter," http://arxiv.org/abs/0806.2036. 

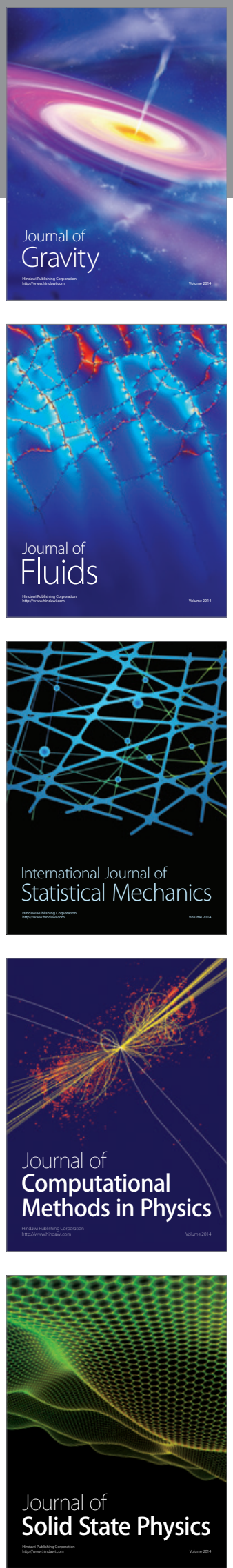

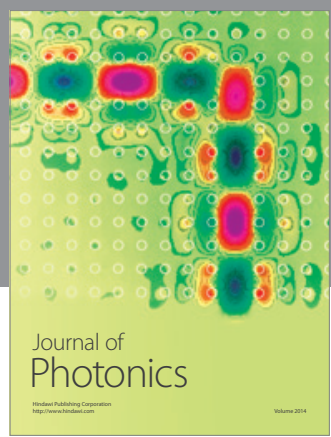

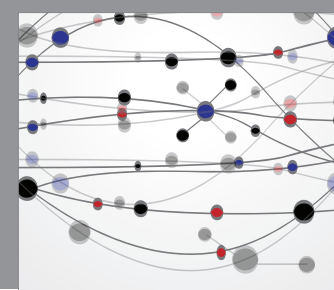

The Scientific World Journal

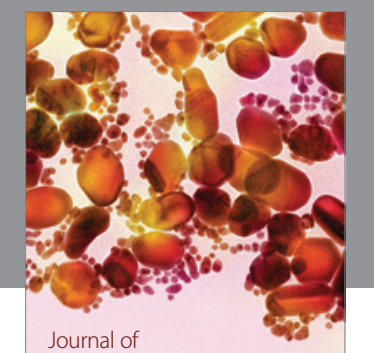

Soft Matter
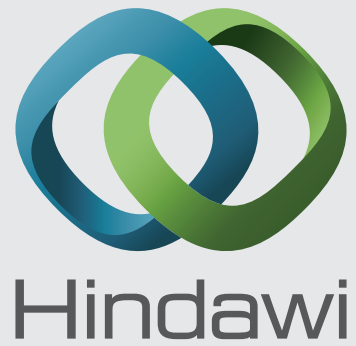

Submit your manuscripts at

http://www.hindawi.com
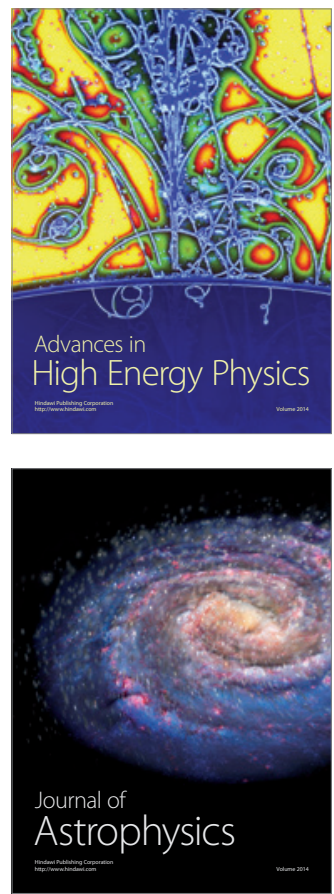
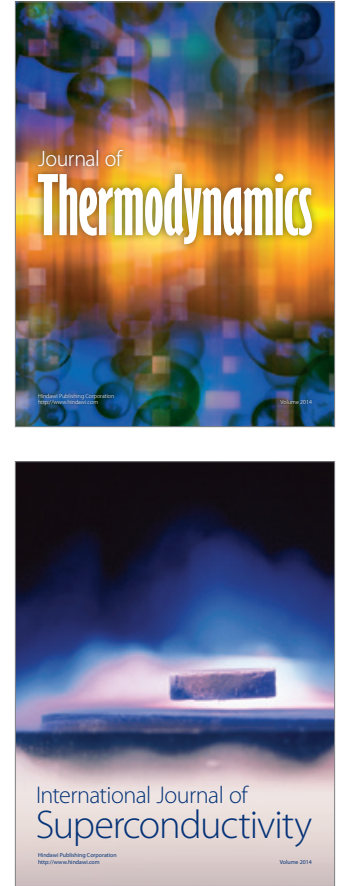
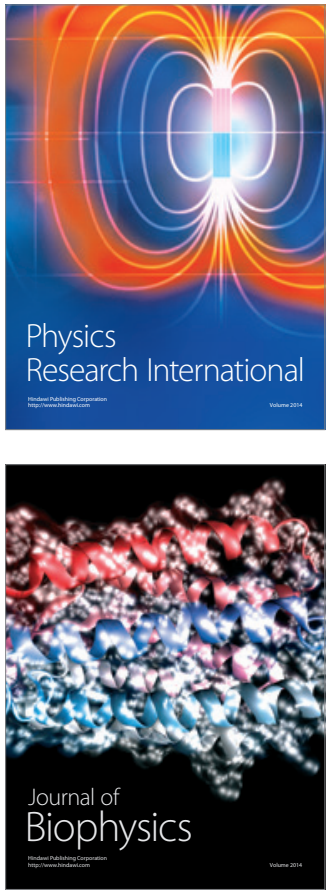
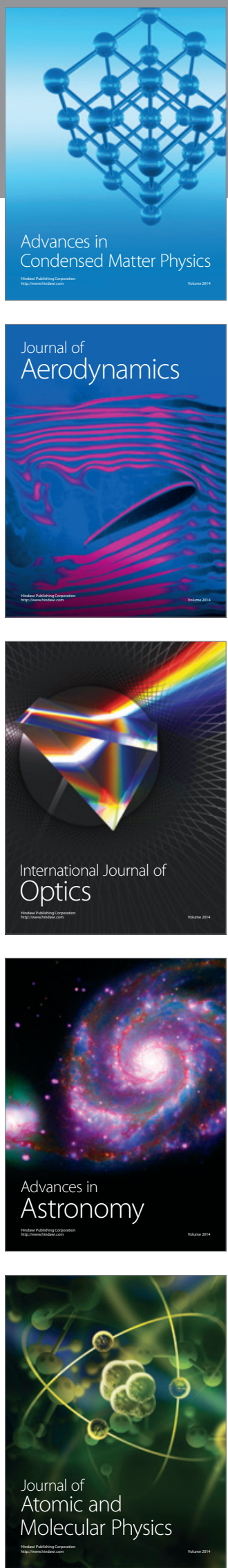